KODAI MATH. SEM. REP.

20 (1968), 414-436

\title{
ON CERTAIN OPERATORS ASSOCIATED WITH TENSOR FIELDS
}

\author{
By Kentaro Yano and Mitsue Ako
}

Dedicated to Professor Shisanji Hokari on his sixtieth birthday

\section{Introduction.}

The theory of differential concomitants has been developed by Frölicher, Nijenhuis [1], [2], [6], Schouten [9] and others. ${ }^{1)}$

Schouten [9] introduced a concomitant $[P, Q]$ formed with tensor fields $P$ and $Q$ of type $(p+1,0)$ and $(q+1,0)$ respectively, which is a tensor field of type $(p+q+1,0)$.

Nijenhuis [6] introduced a concomitant $[S, T]$ formed with a vector $s$-form $S$ and a vector $t$-form $T$ which is a vector $(s+t)$-form. Frölicher and Nijenhuis [1], [2], also introduced a concomitant $[S, \omega]$ formed with a vector $s$-form $S$ and a scalar $t$-form $\omega$ which is a scalar $(s+t)$-form.

On the other hand it was found that, in the study of a differentiable manifold $M$ with an almost complex structure $F$, the tensor

$$
\begin{aligned}
N(X, Y) & =\frac{1}{2}[F, F](X, Y) \\
& =[F X, F Y]-F[X, F Y]-F[F X, Y]+F^{2}[X, Y]
\end{aligned}
$$

introduced by Nijenhuis [5] plays an important part. It is now well known [4] that it is necessary and sufficient for an almost complex manifold to be complex that the Nijenhuis tensor $N$ formed with $F$ vanishes identically.

Nijenhuis [6] proved also that

$$
[F, N]=0, \quad[N, N]=0
$$

and it seemed that there is no concomitant formed only with $F$ and its partial derivatives and being essentially distinct from $N$.

However Walker [13] found a tensor field of type $(1,4)$ involving the second partial derivatives of an almost complex structure $F$.

Willmore [14] introduced a tensor field $W$ of type $(1,4)$

$$
W=[F \pi N, N]=[F, N \pi N]
$$

Received February 22, 1968.

1) The numbers between brackets refer to the Bibliography at the end of the paper. 
formed with a general tensor field $F$ of type $(1,1)$ and showed that $W$ reduces to the skew-symmetric part of the tensor field introduced by Walker when $F$ defines an almost complex structure of the manifold, where $\pi$ denotes a kind of inner product introduced by Frölicher and Nijenhuis [1].

Recently Ślebodziński [10] introduced a tensor field of type $(1,3)$ formed only with an almost complex structure $F$ and containing the second partial derivatives of $F$, but Willmore [15] proved that the tensor introduced by Slebodziński is identically zero.

Now, in a complex manifold, the fact that the components of a vector field or a 1-form are analytic functions of complex coordinates of the manifold has a geometrical meaning. For example, in a Kählerian manifold, a vector field whose components are analytic functions of complex coordinates is a so-called geodesic vector field [19] and a 1-form whose components are analytic functions of complex coordinates is a harmonic 1-form [16]. We can call them a contravariant analytic vector field and a covariant analytic vector field. For a tensor field, the fact that the components are analytic functions of complex coordinates has a geometrical meaning only when the tensor is pure [16].

The analyticity of a vector field, a 1-form or a pure tensor field can be generalized to the almost analyticity of these geometric objects in an almost complex manifold.

The almost analyticity of a vector field, a 1-form or a pure tensor in an almost complex manifold is defined by the vanishing of certain quantities formed with the components of these geometric objects.

It is known that these quantities are components of differential concomitants formed with the almost complex structure $F$ and these geometric objects.

The main purpose of the present paper is to generalize these differential concomitants and to discuss relations between these and concomitants already known.

In the sequel, we assume that the manifold, the geometric objects such as vector fields, differential forms, and tensor fields are all of differentiability class $C^{\infty}$. We denote by $\mathscr{I}_{s}^{r}(M)$ the sets of tensor fields of contravariant degree $r$ and covariant degree $s$. A function belongs to $\mathscr{I}_{0}^{0}(M)$, a vector field to $\mathscr{I}_{0}^{1}(M)$ or briefly to $\mathscr{I}^{1}(M)$ and a 1 -form to $\mathscr{I}_{1}^{0}(M)$ or briefly to $\mathscr{I}_{1}(M)$. We use also the notation

$$
\left.S_{[i} p^{i} p-1 \cdots i_{1}\right]
$$

to denote the so-called skew-symmetric part of $S_{i_{p} i_{p-1} \cdots i_{1}}$ (See, e.g. [9]).

\section{$\S 1$. Operator $\Phi^{F}(X)$ applied to a vector field.}

We first define an operator $\Phi^{F}(X)$ associated with a given $F \in \mathscr{I}_{1}^{1}(M)$ and an arbitrary $X \in \mathscr{I}_{0}^{1}(M)$ and applied to an arbitrary $Y \in \mathscr{I}_{0}^{1}(M)$ by

$$
\Phi^{F}(X) Y=-\left(\mathcal{L}_{Y} F\right) X,
$$


that is,

$$
\Phi^{F}(X) Y=[F X, Y]-F[X, Y] .
$$

We see that $\Phi^{F}(X) Y$ is linear in $X$, but not in $Y$. We have in fact

$$
\Phi^{F}(X)(g Y)=g\left\{\Phi^{F}(X) Y\right\}+((F X) g) Y-(X g) F Y
$$

for any $g \in \mathscr{I}_{0}^{\circ}(M)$.

When $F$ defines an almost complex structure in $\dot{M}$, that is, when $F$ satisfies $F^{2}=-I, I$ denoting the unit tensor, a vector field $Y$ which satisfies $\mathcal{L}_{Y} F=0$ is said to be almost contravariant analytic. Thus a necessary and sufficient condition that a vector field $Y$ in a manifold $M$ with almost complex structure $F$ is almost contravariant analytic is $\Phi^{F}(X) Y=0$ for any $X \in \mathscr{I}_{0}^{1}(M)$.

We often write $\Phi(X)$ for $\Phi^{F}(X)$ in the sequel when it is clear that it is associated with $F$.

\section{§ 2. Operator $\Phi^{F}(X)$ applied to a tensor field of type $(1,1)$.}

Let $G \in \mathscr{I}_{1}^{1}(M)$. We consider the expression

$$
\Phi(X)(G Y)-G(\Phi(X) Y)=\left(-\mathcal{L}_{G Y} F+G \mathcal{L}_{Y} F\right) X
$$

that is,

(2. 2) $\Phi(X)(G Y)-G(\Phi(X) Y)=[F X, G Y]-F[X, G Y]-G[F X, Y]+G F[X, Y]$,

where $X, Y \in \mathscr{I}_{0}^{1}(M)$. By the definition, (2.2) is linear in $X$, but not in $Y$ in general. We have in fact

(2. 3) $\Phi(X)(G(g Y))-G(\Phi(X)(g Y))=g\{\Phi(X)(G Y)-G(\Phi(X) Y)\}-(X g)(F G-G F) Y$, for $g \in \mathscr{I}_{0}^{0}(M)$. Thus we have

Proposition 2.1. Let $G \in \mathscr{I}_{1}^{1}(M)$. The expression (2.2) is linear in $X$ and $Y$ and defines a tensor field $\in \mathscr{I}_{2}^{1}(M)$ if and only if $F G=G F$.

Thus, if the condition $F G=G F$ is satisfied, we put

$$
\Phi(X)(G Y)-G(\Phi(X) Y)=(\Phi(X) G) Y=(\Phi G)(X, Y) .
$$

Since $F G=G F$ is trivially satisfied for $F=G$, we have

Corollary 2.1. $(\Phi(X) F) Y$ defines a tensor field $\in \mathscr{I}_{2}^{1}(M)$.

Since

$$
\begin{aligned}
(\Phi(X) F) Y & =\Phi(X)(F Y)-F(\Phi(X) Y) \\
& =[F X, F Y]-F[X, F Y]-F[F X, Y]+F^{2}[X, Y]
\end{aligned}
$$


$(\Phi(X) F) Y$ is nothing but the Nijenhuis tensor $N(X, Y)$ constructed from $F$ :

$$
(\Phi(X) F) Y=N(X, Y) \text {. }
$$

When $F$ is an almost complex structure in $M$, a tensor field $G \in \mathscr{I}_{1}^{1}(M)$ which satisfies $F G=G F$ is said to be pure. Thus we have

CoRollary 2.2. If $G$ is a pure tensor $\in \mathscr{I}_{1}^{1}(M)$ in a manifold $M$ with almost complex structure $F$, then $(\Phi(X) G) Y$ defines a tensor field $\in \mathscr{I}_{2}^{1}(M)$.

When $(\Phi(X) G) Y=0$ for a pure tensor $G \in \mathscr{I}_{1}^{1}(M)$ and for any $X, Y \in \mathscr{I}_{0}^{1}(M), M$ being a manifold with almost complex structure $F, G$ is said to be almost analytic.

Equation (2.3) shows that

$$
\Phi^{F}(X)(G Y)-G\left(\Phi^{F}(X) Y\right)+\Phi^{G}(X)(F Y)-F\left(\Phi^{G}(X) Y\right)
$$

is linear in $X$ and $Y$. Thus we have

Proposition 2.2. Let $F, G \in \mathscr{I}_{1}^{1}(M)$. The expression (2.6) defines a tensor field $\in \mathscr{I}_{2}^{1}(M)$.

Since

$$
\begin{aligned}
& \Phi^{F}(X)(G Y)-G\left(\Phi^{F}(X) Y\right)+\Phi^{G}(X)(F Y)-F\left(\Phi^{G}(X) Y\right) \\
= & {[F X, G Y]-F[X, G Y]-G[F X, Y]+G F[X, Y] } \\
& +[G X, F Y]-G[X, F Y]-F[G X, Y]+F G[X, Y],
\end{aligned}
$$

the expression (2.6) is nothing but the torsion $[F, G](X, Y)$ of $F$ and $G$ :

$$
\Phi^{F}(X)(G Y)-G\left(\Phi^{F}(X) Y\right)+\Phi^{a}(X)(F Y)-F\left(\Phi^{a}(X) Y\right)
$$

$$
=[F, G](X, Y) \text {. }
$$

On the other hand, we have from (2.3)

$$
\begin{aligned}
& \Phi(f X)(G(g Y))-G(\Phi(f X)(g Y))-\Phi(g Y)(G(f X))+G(\Phi(g Y)(f X)) \\
= & f g\{\Phi(X)(G Y)-G(\Phi(X) Y)-\Phi(Y)(G X)+G(\Phi(Y) X)\}-(F G-G F)(f(X g) Y-g(Y f) X) .
\end{aligned}
$$

This equation and

$$
[f X, g Y]=f g[X, Y]+f(X g) Y-g(Y f) X
$$

show that

$$
\Phi(X)(G Y)-G(\Phi(X) Y)-\Phi(Y)(G X)+G(\Phi(Y) X)+(F G-G F)[X, Y]
$$

is linear in $X$ and $Y$. This also coincides with the torsion $[F, G](X, Y)$ of $F$ and $G$. 
Remark 2.1. Let $X^{h}, Y^{h}, F_{i}{ }^{h}$ and $G_{i}{ }^{h}$ be components of $X, Y, F$ and $G$ respectively with respect to a natural coordinate system in $M$. Then the expression (2.1) has components

$$
\left(F_{\jmath}{ }^{t} \partial_{t} G_{i}{ }^{h}-G_{i}{ }^{t} \partial_{t} F_{j}{ }^{h}+G_{a}{ }^{h} \partial_{i} F_{\jmath}{ }^{a}-F_{a}{ }^{h} \partial_{j} G_{i}{ }^{a}\right) X^{j} Y^{i}-\left(F_{a}{ }^{h} G_{i}{ }^{a}-G_{a}{ }^{h} F_{i}^{a}\right)\left(X^{t} \partial_{t} Y^{i}\right) .
$$

This equation shows, as Proposition 2.1 says, that

$$
(F, G)_{j i}{ }^{h}=F_{j}{ }^{t} \partial_{t} G_{i}{ }^{h}-G_{i}{ }^{t} \partial_{t} F_{j}{ }^{h}+G_{a}{ }^{h} \partial_{i} F_{j}{ }^{a}-F_{a}{ }^{h} \partial_{j} G_{i}{ }^{a}
$$

are components of a tensor field of type $(1,2)$ when the condition $F G=G F$ is satisfied.

But (2.9) shows also that

$$
\left\{(F, G)_{j i}{ }^{h}-(F, G)_{i j}{ }^{h}\right\} X^{j} Y^{i}-\left(F_{a}{ }^{h} G_{i}{ }^{a}-G_{a}{ }^{h} F_{\imath}{ }^{a}\right)[X, Y]^{i}
$$

are components of a vector field for any $X^{h}$ and $Y^{h}$ and consequently

$$
(F, G)_{j i}^{h}-(F, G)_{i j}{ }^{h}
$$

are components of a tensor field of type $(1,2)$, skew-symmetric in $j$ and $i$, without the condition $F G=G F$. This coincides with the torsion tensor $[F, G]$ :

$$
(F, G)-(G, F)=[F, G] \text {. }
$$

\section{§ 3. Operator $\Phi^{F}(X)$ applied to a tensor field of type $(1,2)$.}

Let $S \in \mathscr{I}_{2}^{1}(M)$. We consider the expression

$$
\begin{aligned}
& \Phi(X)(S(Y, Z))-S(\Phi(X) Y, Z)-S(Y, \Phi(X) Z) \\
= & -\left(\mathcal{L}_{S(Y, Z)} F\right) X+S\left(\left(\mathcal{L}_{Y} F\right) X, Z\right)+S\left(Y,\left(\mathcal{L}_{Z} F\right) X\right),
\end{aligned}
$$

that is,

$$
\begin{aligned}
& \Phi(X)(S(Y, Z))-S(\Phi(X) Y, Z)-S(Y, \Phi(X) Z) \\
= & {[F X, S(Y, Z)]-F[X, S(Y, Z)]-S([F X, Y]-F[X, Y], Z)-S(Y,[F X, Z]-F[X, Z]) }
\end{aligned}
$$

for any $X, Y, Z \in \mathscr{I}_{0}^{1}(M)$.

By the definition, (3.1) is linear in $X$, but not in $Y$ and $Z$ in general. We have in fact

$$
\begin{aligned}
& \Phi(X)(S(g Y, h Z))-S(\Phi(X)(g Y), h Z)-S(g Y, \Phi(X)(h Z)) \\
= & g h\{\Phi(X)(S(Y, Z))-S(\Phi(X) Y, Z)-S(Y, \Phi(X) Z)\} \\
& -(X(g h)) F(S(Y, Z))+(X g) h S(F Y, Z)+g(X h) S(Y, F Z)
\end{aligned}
$$

for any $g, h \in \mathscr{I}_{0}^{\circ}(M)$. Thus we have 
Proposition 3.1. Let $S \in \mathscr{I}_{2}^{1}(M)$. The expression (3.1) is linear in $X, Y$ and $Z$ and defines a tensor field $\in \mathscr{I}_{3}^{1}(M)$ if and only if $S$ satisfies

$$
F(S(Y, Z))=S(F Y, Z)=S(Y, F Z)
$$

for any $Y, Z \in \mathscr{I}_{0}^{1}(M)$.

Thus if the condition (3.4) is satisfied, we put

$$
\begin{aligned}
\Phi(X)(S(Y, Z)) & -S(\Phi(X) Y, Z)-S(Y, \Phi(X) Z) \\
& =(\Phi(X) S)(Y, Z) \\
& =(\Phi S)(X, Y, Z) .
\end{aligned}
$$

When $F$ is an almost complex structure in $M$, an $S \in \mathscr{I}_{2}^{1}(M)$ which satisfies (3.4) is said to be pure. Thus we have

COROllary 3.1. If $S$ is a pure tensor $\in \mathscr{I}_{2}^{1}(M)$ in $M$ with almost complex structure $F$, (3.1) defines a tensor field $\in \mathscr{I}_{3}^{1}(M)$.

When (3. 1) vanishes for a pure tensor $S \in \mathscr{I}_{2}^{1}(M)$ and for any $X, Y, Z \in \mathscr{I}_{0}^{1}(M)$ in $M$ with almost complex structure $F, S$ is said to be almost analytic.

On the other hand, we have from (3.3)

$$
\begin{gathered}
\Phi(f X)(S(g Y, h Z)-S(\Phi(f X)(g Y), h Z)-S(g Y, \Phi(f X)(h Z)) \\
+\Phi(g Y)(S(h Z, f X))-S(\Phi(g Y)(h Z), f X)-S(h Z, \Phi(g Y)(f X)) \\
+\Phi(h Z)(S(f X, g Y))-S(\Phi(h Z)(f X), g Y)-S(f X, \Phi(h Z)(g Y)) \\
=f g h\{\Phi(X)(S(Y, Z))-S(\Phi(X) Y, Z)-S(Y, \Phi(X) Z) \\
\quad+\Phi(Y)(S(Z, X))-S(\Phi(Y) Z, X)-S(Z, \Phi(Y) X) \\
\quad+\Phi(Z)(S(X, Y))-S(\Phi(Z) X, Y)-S(X, \Phi(Z) Y)\} \\
-(f X(g h)) F(S(Y, Z))+f h(X g) S(F Y, Z)+f g(X h) S(Y, F Z) \\
-(g Y(h f)) F(S(Z, X))+g f(Y h) S(F Z, X)+g h(Y f) S(Z, F X) \\
-(h Z(f g)) F(S(X, Y))+h g(Z f) S(F X, Y)+h f(Z g) S(X, F Y) .
\end{gathered}
$$

This equation and

$$
\begin{aligned}
& F(S([f X, g Y], h Z))+F(S([g Y, h Z], f X))+F(S([h Z, f X], g Y)) \\
& -S(F[f X, g Y], h Z)-S(F[g Y, h Z], f X)-S(F[h Z, f X], g Y) \\
= & f g h\{F(S([X, Y], Z))+F(S([Y, Z], X))+F(S([X, Z], Y)) \\
& -S(F[X, Y], Z)-S(F[Y, Z], X)-S(F[Z, X], Y)\}
\end{aligned}
$$




$$
\begin{aligned}
& +(f X(g h)) F(S(Y, Z))+(g Y(h f)) F(S(Z, X))+(h Z(f g)) F(S(X, Y)) \\
& -f h(X g) S(F Y, Z)-g f(Y h) S(F Z, X)-h g(Z f) S(F X, Y) \\
& +g h(Y f) S(F X, Z)+h f(Z g) S(F Y, X)+f g(X h) S(F Z, Y)
\end{aligned}
$$

give

Proposition 3. 2. The expression

$$
\begin{aligned}
& C(X, Y, Z)[\Phi(X)(S(Y, Z))-S(\Phi(X) Y, Z)-S(Y, \Phi(X) Z) \\
& \left.\quad+\frac{1}{2}\{F(S([X, Y], Z))+F(S(Y,[X, Z]))-S(F[X, Y], Z)-S(Y, F[X, Z])\}\right]
\end{aligned}
$$

is linear in $X, Y, Z$ and defines a tensor field $\in \mathscr{I}_{3}^{1}(M)$, where $C(X, Y, Z)$ denotes the sum of terms obtained by applying a cyclic permutation of $X, Y$ and $Z$.

The actual expression for (3. 8) is

$$
\begin{aligned}
& {[F X, S(Y, Z)]-F[X, S(Y, Z)]-S([F X, Y]-F[X, Y], Z)-S(Y,[F X, Z]-F[X, Z])} \\
& \quad+[F Y, S(Z, X)]-F[Y, S(Z, X)]-S([F Y, Z]-F[Y, Z], X)-S(Z,[F Y, X]-F[Y, Z]) \\
& \quad+[F Z, S(X, Y)]-F[Z, S(X, Y)]-S([F Z, X]-F[Z, X], Y)-S(X,[F Z, Y]-F[Z, Y]) \\
& \quad+F(S([X, Y], Z))+F(S([Y, Z], X))+F(S([Z, X], Y)) \\
& \quad-S(F[X, Y], Z)-S(F[Y, Z], X)-S(F[Z, X], Y),
\end{aligned}
$$

if $S$ is skew-symmetric.

We can check by an elementary but rather tedious tensor computation that, if $S(Y, Z)$ is the Nijenhuis tensor

$$
S(Y, Z)=[F Y, F Z]-F[Y, F Z]-F[F Y, Z]+F^{2}[Y, Z]
$$

then the expression (3.8) is identically zero.

Thus

Proposition 3. 3. The expression (3.8) is identically zero for $F$ and $S(X, Y)$ $=N(X, Y)$.

REMARK 3. 1. Let $X^{h}, Y^{h}, Z^{h}, F_{i}^{h}$ and $S_{j i}^{h}$ be components of $X, Y, Z, F$ and $S$ respectively with respect to a natural coordinate system in $M$. Then the expression (3.2) has components

$$
\begin{aligned}
& \left(F_{k}^{a} \partial_{a} S_{j i}{ }^{h}-F_{a}{ }^{h} \partial_{k} S_{j i}{ }^{a}-S_{j i}{ }^{a} \partial_{a} F_{k}{ }^{h}+S_{a i}{ }^{h} \partial_{j} F_{k}{ }^{a}+S_{j a}{ }^{h} \partial_{i} F_{k}{ }^{a}\right) X^{k} Y^{j} Z^{\imath} \\
& \quad+\left(F_{j}{ }^{a} S_{a i}{ }^{h}-F_{a}{ }^{h} S_{j i}{ }^{a}\right)\left(X^{k} \partial_{k} Y^{j}\right) Z^{\imath}+\left(F_{\imath}{ }^{a} S_{j a}{ }^{h}-F_{a}{ }^{h} S_{j i}{ }^{a}\right) Y^{j}\left(X^{k} \partial_{k} Z^{i}\right) .
\end{aligned}
$$

Thus (3.9) shows, as Proposition 3.1 says, that 


$$
(F, S)_{k j i}^{h}=F_{k}^{a} \partial_{a} S_{j i}{ }^{h}-F_{a}{ }^{h} \partial_{k} S_{j i}{ }^{a}-S_{j i}{ }^{a} \partial_{a} F_{k}{ }^{h}+S_{a i}{ }^{h} \partial_{j} F_{k}^{a}+S_{j a}{ }^{h} \partial_{i} F_{k}{ }^{a}
$$

are components of a tensor field of type $(1,3)$ when the conditions

$$
F_{a}{ }^{h} S_{j i}{ }^{a}=F_{\jmath}{ }^{a} S_{a i}{ }^{h}=F_{\imath}{ }^{a} S_{j a}{ }^{h}
$$

are satisfied.

On the other hand, (3.9) shows that,

$$
3(F, S)_{[k j i]^{h}} X^{k} Y^{j} Z^{i}+\left(F_{\jmath}^{a} S_{[a i]}{ }^{h}-F_{a}{ }^{h} S_{[j i]}{ }^{a}\right)\left([X, Y]^{j} Z^{\imath}+[Y, Z]^{j} X^{\imath}+[Z, X]^{j} Y^{i}\right)
$$

and consequently

$$
(F, S)_{[k j i]}^{h}
$$

are components of a tensor field of type $(1,3)$, skew-symmetric in $k, j$ and $i$, without the condition (3.4). For a skew-symmetric tensor $S$, this tensor is equal to $[F, S]$ introduced by Nijenhuis and was proved to be zero when $S$ is the Nijenhuis tensor formed with $F$ ([6]).

The expression (3.11) can be written as

$$
\begin{aligned}
& \left(F_{k}{ }^{a} \partial_{a} S_{j i}{ }^{h}+F_{\jmath}{ }^{a} \partial_{a} S_{i k}{ }^{h}+F_{\imath}{ }^{a} \partial_{a} S_{k j}{ }^{h}\right)-F_{a}^{h}\left(\partial_{k} S_{j i}{ }^{a}+\partial_{\jmath} S_{i k}{ }^{a}+\partial_{i} S_{k \jmath}{ }^{a}\right) \\
& -\left(S_{k j}{ }^{a} \partial_{a} F_{i}^{h}+S_{j i}^{a} \partial_{a} F_{k}{ }^{h}+S_{i k}{ }^{a} \partial_{a} F_{j}{ }^{h}\right) \\
& +S_{k a}{ }^{h}\left(\partial_{j} F_{\imath}^{a}-\partial_{i} F_{\jmath}{ }^{a}\right)+S_{\jmath a}{ }^{h}\left(\partial_{i} F_{k}{ }^{a}-\partial_{k} F_{\imath}{ }^{a}\right)+S_{i a}{ }^{h}\left(\partial_{k} F_{\jmath}{ }^{a}-\partial_{j} F_{k}^{a}\right),
\end{aligned}
$$

when $S$ is skew-symmetric.

As Willmore [15] showed it, this reduces to the tensor introduced by Ślebodziński ([10]) when $F$ is an almost complex structure and $S$ is the Nijenhuis tensor formed with $F$, and hence the Ślebodziński tensor is identically zero.

\section{§4. Operator $\Phi^{F}(X)$ applied to a tensor field of type $(1, s)$.}

Let $T \in \mathscr{I}_{s}^{1}(M)$. We consider the expression

$$
\begin{aligned}
& \Phi(X)\left(T\left(Y_{1}, Y_{2}, \cdots, Y_{s}\right)\right)-T\left(\Phi(X) Y_{1}, Y_{2}, \cdots, Y_{s}\right)-\cdots-T\left(Y_{1}, Y_{2}, \cdots, \Phi(X) Y_{s}\right) \\
= & -\left(\mathcal{L}_{T\left(Y_{1}, Y_{2}, \cdots, Y_{s}\right)} F\right) X+T\left(\left(\mathcal{L}_{Y_{1}} F\right) X, Y_{2}, \cdots, Y_{s}\right)+\cdots+T\left(Y_{1}, Y_{2}, \cdots,\left(\mathcal{L}_{Y_{s}} F\right) X\right),
\end{aligned}
$$

that is,

$$
\begin{aligned}
& \Phi(X)\left(T\left(Y_{1}, Y_{2}, \cdots, Y_{s}\right)\right)-T\left(\Phi(X) Y_{1}, Y_{2}, \cdots, Y_{s}\right)-\cdots-T\left(Y_{1}, Y_{2}, \cdots, \Phi(X) Y_{s}\right) \\
= & {\left[F X, T\left(Y_{1}, Y_{2}, \cdots, Y_{s}\right)\right]-F\left[X, T\left(Y_{1}, Y_{2}, \cdots, Y_{s}\right)\right] } \\
& -T\left(\left[F X, Y_{1}\right], Y_{2}, \cdots, Y_{s}\right)+T\left(F\left[X, Y_{1}\right], Y_{2}, \cdots, Y_{s}\right) \\
& -\cdots-T\left(Y_{1}, Y_{2}, \cdots,\left[F X, Y_{s}\right]\right)+T\left(Y_{1}, Y_{2}, \cdots, F\left[X, Y_{s}\right]\right)
\end{aligned}
$$


for $X, Y_{1}, Y_{2}, \cdots, Y_{s} \in \mathscr{I}_{0}^{1}(M)$.

By the definition, (4.2) is linear in $X$, but not in $Y_{1}, Y_{2}, \cdots, Y_{s}$ in general. We have in fact

$$
\begin{aligned}
& \Phi(X)\left(T\left(g_{1} Y_{1}, g_{2} Y_{2}, \cdots, g_{s} Y_{s}\right)\right)-T\left(\Phi(X)\left(g_{1} Y_{1}\right), g_{2} Y_{2}, \cdots, g_{s} Y_{s}\right) \\
& \quad-\cdots-T\left(g_{1} Y_{1}, g_{2} Y_{2}, \cdots, \Phi(X)\left(g_{s} Y_{s}\right)\right) \\
& =\left(g_{1} g_{2} \cdots g_{s}\right)\left\{\Phi(X)\left(T\left(Y_{1}, Y_{2}, \cdots, Y_{s}\right)\right)-T\left(\Phi(X) Y_{1}, Y_{2}, \cdots, Y_{s}\right)\right. \\
& \left.\quad-\cdots-T\left(Y_{1}, Y_{2}, \cdots, \Phi(X) Y_{s}\right)\right\} \\
& \quad-\left(X\left(g_{1} g_{2} \cdots g_{s}\right)\right) F\left(T\left(Y_{1}, Y_{2}, \cdots, Y_{s}\right)\right)+\left(X g_{1}\right) g_{2} \cdots g_{s} T\left(F Y_{1}, Y_{2}, \cdots, Y_{s}\right) \\
& +g_{1}\left(X g_{2}\right) \cdots g_{s} T\left(Y_{1}, F Y_{2}, \cdots, Y_{s}\right)+\cdots+g_{1} g_{2} \cdots\left(X g_{s}\right) T\left(Y_{1}, Y_{2}, \cdots, F Y_{s}\right)
\end{aligned}
$$

for $g_{1}, g_{2}, \cdots, g_{s} \in \mathscr{I}_{0}^{0}(M)$. Thus we have

Proposition 4.1. Let $T \in \mathscr{I}_{s}^{1}(M)$. The expression (4.2) is linear in $X, Y_{1}, Y_{2}$, $\cdots, Y_{s}$ and defines a tensor field $\in \mathscr{I}^{1}{ }_{s+1}(M)$ if and only if $T$ satisfies

$$
\begin{aligned}
F\left(T\left(Y_{1}, Y_{2}, \cdots, Y_{s}\right)\right) & =T\left(F Y_{1}, Y_{2}, \cdots, Y_{s}\right) \\
& =T\left(Y_{1}, F Y_{2}, \cdots, Y_{s}\right)
\end{aligned}
$$

$$
=T\left(Y_{1}, Y_{2}, \cdots, F Y_{s}\right)
$$

Thus, if (4.4) is satisfied, we put

$$
\begin{aligned}
& \Phi(X)\left(T\left(Y_{1}, Y_{2}, \cdots, Y_{s}\right)\right)-T\left(\Phi(X) Y_{1}, Y_{2}, \cdots, Y_{s}\right)-\cdots-T\left(Y_{1}, Y_{2}, \cdots, \Phi(X) T_{s}\right) \\
= & (\Phi(X) T)\left(Y_{1}, Y_{2}, \cdots, Y_{s}\right) \\
= & (\Phi T)\left(X, Y_{1}, Y_{2}, \cdots, Y_{s}\right) .
\end{aligned}
$$

When $F$ is an almost complex structure in $M$, a $T \in \mathscr{I}_{s}^{1}(M)$ which satisfies (4.4) is said to be pure. Thus we have

CoROLlaRY 4.1. If $T$ is a pure tensor $\in \mathscr{I}_{s}^{1}(M)$ in $M$ with almost complex structure $F,(4.2)$ defines a tensor field $\in \mathscr{I}^{1}{ }_{s+1}(M)$.

When (4.2) vanishes for a pure tensor $T \in \mathscr{I}_{s}^{1}(M)$ for any $X, Y_{1}, Y_{2}, \cdots, Y_{s} \in \mathscr{I}_{0}^{1}(M)$, $T$ is said to be almost analytic (See, for example, [7], [11], [16]).

On the other hand, we have from (4.3)

$$
\begin{aligned}
& C\left(f X, g_{1} Y_{1}, g_{2} Y_{2}, \cdots, g_{s} Y_{s}\right)\left[\Phi(f X)\left(T\left(g_{1} Y_{1}, g_{2} Y_{2}, \cdots, g_{s} Y_{s}\right)\right)\right. \\
& \left.\quad-T\left(\Phi(f X)\left(g_{1} Y_{1}\right), g_{2} Y_{2}, \cdots, g_{s} Y_{s}\right)-\cdots-T\left(g_{1} Y_{1}, g_{2} Y_{2}, \cdots, \Phi(f X)\left(g_{s} Y_{s}\right)\right)\right]
\end{aligned}
$$


OPERATORS ASSOCIATED WITH TENSOR FIELDS

$$
\begin{aligned}
= & C\left(X, Y_{1}, Y_{2}, \cdots, Y_{s}\right)\left[f g _ { 1 } g _ { 2 } \cdots g _ { s } \left\{\Phi(X)\left(T\left(Y_{1}, Y_{2}, \cdots, Y_{s}\right)\right)\right.\right. \\
& \left.\left.-T\left(\Phi(X) Y_{1}, Y_{2}, \cdots, Y_{s}\right)-\cdots-T\left(Y_{1}, Y_{2}, \cdots, \Phi(X) Y_{s}\right)\right\}\right] \\
& -C\left(f X, g_{1} Y_{1}, g_{2} Y_{2}, \cdots, g_{s} Y_{s}\right)\left[\left(f X\left(g_{1} g_{2} \cdots g_{s}\right)\right) F\left(T\left(Y_{1}, Y_{2}, \cdots, Y_{s}\right)\right)\right. \\
& \left.-f\left(X g_{1}\right) g_{2} \cdots g_{s} T\left(F Y_{1}, Y_{2}, \cdots, Y_{s}\right)-\cdots-f g_{1} \cdots X\left(g_{s}\right) T\left(Y_{1}, Y_{2}, \cdots F Y_{s}\right)\right],
\end{aligned}
$$

from which we have

Proposition 4. 2.

$$
\begin{aligned}
C\left(X, Y_{1}, \cdots, Y_{s}\right)\left[\Phi(X)\left(T\left(Y_{1}, Y_{2}, \cdots, Y_{s}\right)\right)\right. & \\
& -T\left(\Phi(X) Y_{1}, Y_{2}, \cdots, Y_{s}\right)-\cdots-T\left(Y_{1}, Y_{2}, \cdots, \Phi(X) Y_{s}\right) \\
& \left.+\frac{1}{2} \sum_{t=1}^{s}\left\{F\left(T\left(Y_{1}, \cdots,\left[X, Y_{t}\right], \cdots, Y_{s}\right)\right)+T\left(T_{1}, \cdots, F\left[X, Y_{t}\right], \cdots, Y_{s}\right)\right\}\right]
\end{aligned}
$$

is linear in $X, Y_{1}, \cdots, Y_{s}$ and defines a tensor field $\in \mathscr{I}_{s+1}^{1}(M)$, where $C\left(X, Y_{1}, \cdots, Y_{s}\right)$ denotes the sum of terms obtained by applying a cyclic permutation of $X, Y_{1}, \cdots, Y_{s}$.

REMARK 4.1. Let $X^{h}, Y_{1}{ }^{n}, \cdots, Y_{s}{ }^{h}, F_{i}{ }^{h}$ and $T_{\jmath_{s} \cdots i_{1}}{ }^{h}$ be components of $X, Y_{1}$, $\cdots, Y_{s}, F$ and $T$ respectively with respect to a natural coordinate system in $M$. Then the expression (4.2) has components

$$
\begin{gathered}
\left(F_{k}{ }^{a} \partial_{a} T_{j_{s} \cdots i_{1}}{ }^{h}-F_{a}{ }^{h} \partial_{k} T_{j_{s} \cdots j_{1}}{ }^{a}-T_{j_{s} \cdots i_{1}}{ }^{a} \partial_{a} F_{k}{ }^{h}\right. \\
\left.+\sum_{t=1}^{s} T_{j_{s} \cdots a \cdots j_{i}}{ }^{h} \partial_{j_{t}} F_{k}{ }^{a}\right) X^{k} Y_{1}^{j s} \cdots Y_{s}^{j_{1}} \\
+\sum_{t=1}^{s}\left(F_{j_{t}}{ }^{a} T_{j_{s} \cdots a \cdots j_{1}}{ }^{h}-F_{a}{ }^{h} T_{j_{s} \cdots j_{1}}{ }^{a}\right) Y_{1}^{j_{s}} \cdots\left(X^{k} \partial_{k} Y_{t}^{j t}\right) \cdots Y_{s}^{j_{1}}
\end{gathered}
$$

Thus (4.7) shows, as Proposition 4.1 says, that

$$
\begin{aligned}
& (F, T)_{k_{j_{s} \ldots j_{1}}{ }^{h}} \\
= & F_{k}{ }^{a} \partial_{a} T_{j_{s} \cdots j_{1}}{ }^{h}-F_{a}{ }^{h} \partial_{k} T_{j_{s} \cdots j_{1}}{ }^{a}-T_{j_{s} \cdots j_{1}}{ }^{a} \partial_{a} F_{k}{ }^{h}+\sum_{t=1}^{s} T_{j_{s} \ldots a \ldots j_{1}}{ }^{h} \partial_{j_{t}} F_{k}{ }^{a}
\end{aligned}
$$

are components of a tensor field of type $(1, s+1)$ when the conditions

$$
F_{a}{ }^{h} T_{\jmath_{s} \cdots j_{1}}{ }^{a}=F_{j_{t}}{ }^{a} T_{j_{s} \cdots a \cdots j_{1}}{ }^{h} \quad(t=1,2, \cdots, s)
$$

are satisfied.

On the other hand, (4.7) shows that

$$
\begin{aligned}
& (s+1)(F, T)_{\left[k j_{s} \cdots j_{1}\right]}{ }^{h} \\
& +\frac{1}{2} \sum_{t=1}^{s}\left(F_{\left[\jmath_{t}\right.}{ }^{a} T_{\left.j_{s} \cdots \mid a \cdots j_{1}\right]}{ }^{h}-F_{a}{ }^{h} T_{\left[j_{s} \cdots j_{1}\right]}{ }^{a}\right)\left(Y_{1}^{j s} \cdots\left[X, Y_{t}\right]^{j_{t}} \cdots Y_{s}^{j_{1}}\right. \\
& \left.+\sum_{t \neq u} Y_{1}{ }^{j s} \cdots\left[Y_{u}, Y_{t}\right]^{j_{t}} \cdots Y_{1}^{{ }^{1} 1}\right)
\end{aligned}
$$


and consequently, we see that

$$
(s+1)(F, T)_{\left[k j_{s} \cdots j_{1}\right]^{h}}
$$

are components of a tensor field of type $(1, s+1)$, skew-symmetric in all covariant indices without the condition (4.4).

We can easily verify that (4.9) coincides with $[F, T]$ introduced by Nijenhuis ([6]), when $T$ is skew-symmetric.

\section{$\S 5$. Operator $\Phi(X)$ applied to a 1-form.}

Let $\omega \in \mathscr{I}_{1}^{0}(M)$. We consider the expression

$$
\begin{aligned}
& (F X)(\omega(Y))-X(\omega(F Y))-\omega(\Phi(X) Y) \\
= & \left(\mathcal{L}_{F X} \omega-\mathcal{L}_{X}(\omega \circ F)\right)(Y),
\end{aligned}
$$

where $X, Y \in \mathscr{I}_{0}^{1}(M)$ and the 1 -form $\omega \circ F$ is defined by

$$
(\omega \circ F)(Y)=\omega(F Y)
$$

for any $Y \in \mathscr{I}_{0}^{1}(M)$.

The expression (5.1) shows that the 1-form

$$
\Phi(X) \omega=\mathcal{L}_{F X} \omega-\mathcal{L}_{X}(\omega \circ F)
$$

is linear in $X$ and consequently we have

Proposition 5.1. Let $\omega \in \mathscr{I}_{1}^{0}(M)$. Then the expression (5.2) defines a tensor field of type $(0,2)$.

We shall write

$$
(\Phi(X) \omega)(Y)=(\Phi \omega)(X, Y) .
$$

When $F$ is an almost complex structure in $M$, a 1-form $\omega$ satisfying $\Phi(X) \omega=0$ for any $X \in \mathscr{I}_{0}^{1}(M)$ is said to be almost covariant analytic.

REMARK 5.1. Let $X^{h}, Y^{h}, F_{i}^{h}, \omega_{i}$ be components of $X, Y, F, \omega$ respectively with respect to a certain natural coordinate system in $M$. Then the expression (5.1) has components

$$
\left\{F_{\jmath}{ }^{a} \partial_{a} \omega_{i}-\partial_{j}\left(\omega_{a} F_{\imath}{ }^{a}\right)+\omega_{a} \partial_{i} F_{\jmath}{ }^{a}\right\} X^{j} Y^{\imath} .
$$

This shows, as Proposition 5.1 says, that

$$
(F, \omega)_{j i}=F_{j}{ }^{a} \partial_{a} \omega_{i}-F_{\imath}{ }^{a} \partial_{j} \omega_{a}-\omega_{a}\left(\partial_{j} F_{\imath}^{a}-\partial_{i} F_{j}{ }^{a}\right)
$$

are components of a tensor field of type $(0,2)$.

The $(F, \omega)_{j i}$ is not skew-symmetric in $j$ and $i$ in general. We have 


$$
(F, \omega)_{j i}-(F, \omega)_{i j}=[F, \omega]_{j i}-[I, \omega \circ F]_{j i}
$$

in the notation of Frölicher and Nijenhuis ([1], [2]), $I$ being the unit tensor.

\section{§ 6. Operator $\Phi(X)$ applied to a tensor field of type $(0,2)$.}

Let $\theta \in \mathscr{I}_{2}^{0}(M)$. We consider the expression

$$
\begin{aligned}
& (F X)(\theta(Y, Z))-X(\theta(F Y, Z))-\theta(\Phi(X) Y, Z)-\theta(Y, \Phi(X) Z) \\
= & \left(\mathcal{L}_{F X} \theta-\mathcal{L}_{X}(\theta \circ F)\right)(Y, Z)+\theta\left(Y, F \mathcal{L}_{X} Z\right)-\theta\left(F Y, \mathcal{L}_{X} Z\right),
\end{aligned}
$$

where $X, Y, Z \in \mathscr{I}_{0}^{1}(M)$ and the tensor $\theta \circ F$ is defined by

$$
(\theta \circ F)(Y, Z)=\theta(F Y, Z)
$$

for any $Y, Z \in \mathscr{I}_{0}^{1}(M)$.

By the definition, (6.1) is linear in $X$, but not in $Y$ and $Z$ in general. We have in fact

$$
(F X)(\theta(g Y, h Z))-X(\theta(F(g Y), h Z))-\theta(\Phi(X)(g Y), h Z)-\theta(g Y, \Phi(X)(h Z))
$$

$$
\begin{aligned}
= & g h\{(F X)(\theta(Y, Z))-X(\theta(F Y, Z))-\theta(\Phi(X) Y, Z)-\theta(Y, \Phi(X) Z)\} \\
& -g(X h)(\theta(F Y, Z)-\theta(Y, F Z)),
\end{aligned}
$$

where $g, h \in \mathscr{I}_{0}^{0}(M)$. Thus we have

Proposition 6.1. Let $\theta \in \mathscr{I}_{2}^{0}(M)$. The expression (6.1) is linear in $X, Y$ and $Z$ and defines a tensor field $\in \mathscr{I}_{3}^{\circ}(M)$ if and only if the tensor field $\theta$ satisfies

$$
\theta(F Y, Z)=\theta(Y, F Z)
$$

for any $Y, Z \in \mathscr{I}_{0}^{1}(M)$.

Thus if (6.4) is satisfied, we put

$$
\begin{aligned}
& (F X)(\theta(Y, Z))-X(\theta(F Y, Z))-\theta(\Phi(X) Y, Z)-\theta(Y, \Phi(X) Z) \\
= & (\Phi(X) \theta)(Y, Z)=(\Phi \theta)(X, Y, Z) .
\end{aligned}
$$

When $F$ is an almost complex structure in $M$, a tensor field $\theta \in \mathscr{I}_{2}^{0}(M)$ which satisfies (6.4) is said to be pure. Thus we have

CoRollary 6.1. If $\theta$ is a pure tensor field $\in \mathscr{I}_{2}^{0}(M)$ in $M$ with almost complex structure $F$, then (6.1) defines a tensor field $\in \mathscr{I}_{3}^{\circ}(M)$.

When this tensor vanishes, $\theta$ is said to be almost analytic.

On the other hand, (6.3) shows that 


$$
\begin{gathered}
C(X, Y, Z)[(F X)(\theta(Y, Z))-X(\theta(F Y, Z))-\theta(\Phi(X) Y, Z)-\theta(Y, \Phi(X) Z) \\
\left.+\frac{1}{2}\{\theta(Y, F[X, Z])-\theta(F Y,[X, Z])\}\right]
\end{gathered}
$$

is linear in $X, Y, Z$ and defines a tensor field of type $(0,3)$, where $C(X, Y, Z)$ denotes the sum of terms obtained by applying a cyclic permutation of $X, Y, Z$.

REMARK 6.1. Let $X^{h}, Y^{h}, Z^{h}, F_{i}{ }^{h}$ and $\theta_{j i}$ be components of $X, Y, Z, F$ and $\theta$ respectively with respect to a natural coordinate system in $M$. Then the expression (6.1) has components

$$
\begin{gathered}
\left\{F_{k}{ }^{a} \partial_{a} \theta_{j i}-\partial_{k}\left(\theta_{a i} F_{\jmath}{ }^{a}\right)+\theta_{\jmath a} \partial_{i} F_{k}^{a}+\theta_{a i} \partial_{j} F_{k}^{a}\right\} X^{k} Y^{j} Z^{\imath} \\
+\left(\theta_{\jmath a} F_{i}^{a}-\theta_{a i} F_{j}^{a}\right) X^{k} Y^{j} \partial_{k} Z^{i} .
\end{gathered}
$$

The equation (6.6) shows, as Proposition 6.1 says, that

$$
(F, \theta)_{k j i}=F_{k}{ }^{a} \partial_{a} \theta_{j i}-\partial_{k}\left(\theta_{a i} F_{j}{ }^{a}\right)+\theta_{j a} \partial_{i} F_{k}{ }^{a}+\theta_{a i} \partial_{j} F_{k}{ }^{a}
$$

are components of a tensor field of type $(0,3)$ when the condition

$$
\theta_{\jmath} F_{\imath}^{a}=\theta_{a i} F_{\jmath}{ }^{a}
$$

is satisfied.

But (6.6) shows also that

$$
(F, \theta)_{[k j i]}
$$

are components of a tensor field of type $(0,3)$, skew-symmetric in $k, j, i$ without the condition (6.4).

If the tensor $\theta$ is a 2 -form, then the tensor above coincides with that introduced by Frölicher and Nijenhuis ([1]). In their notation, it is

$$
3(F, \theta)_{[k j i]}=[F, \theta]_{k j i}-[I, \theta \circ F]_{k j i} .
$$

$\S 7$. Operator $\Phi(X)$ applied to a tensor field of type $(0, s)$.

Let $\pi \in \mathscr{I}_{s}^{0}(M)$. We consider the expression

$$
\begin{aligned}
& (F X)\left(\pi\left(Y_{1}, Y_{2}, \cdots, Y_{s}\right)\right)-X\left(\pi\left(F Y_{1}, Y_{2}, \cdots, Y_{s}\right)\right) \\
& \left.\quad-\pi\left(\Phi(X) Y_{1}, Y_{2}, \cdots, Y_{s}\right)\right)-\cdots-\pi\left(Y_{1}, Y_{2}, \cdots, \Phi(X) Y_{s}\right) \\
= & \left(\mathcal{L}_{F X} \pi-\mathcal{L}_{X}(\pi \circ F)\right)\left(Y_{1}, Y_{2}, \cdots, Y_{s}\right) \\
& +\pi\left(Y_{1}, F \mathcal{L}_{X} Y_{2}, \cdots, Y_{s}\right)+\cdots+\pi\left(Y_{1}, Y_{2}, \cdots, F \mathcal{L}_{X} Y_{s}\right) \\
& -\pi\left(F Y_{1}, \mathcal{L}_{X} Y_{2}, \cdots, Y_{s}\right)-\cdots-\pi\left(F Y_{1}, Y_{2}, \cdots, \mathcal{L}_{X} Y_{s}\right) .
\end{aligned}
$$


By the definition, (7.1) is linear in $X$, but not in $Y_{1}, Y_{2}, \cdots, Y_{s}$ in general. We have in fact

$$
\begin{gathered}
(F X)\left(\pi\left(g_{1} Y_{1}, g_{2} Y_{2}, \cdots, g_{s} Y_{s}\right)\right)-X\left(\pi\left(F\left(g_{1} Y_{1}\right), g_{2} Y_{2}, \cdots, g_{s} Y_{s}\right)\right) \\
-\pi\left(\Phi(X)\left(g_{1} Y_{1}\right), g_{2} Y_{2}, \cdots, g_{s} Y_{s}\right)-\cdots-\pi\left(g_{1} Y_{1}, g_{2} Y_{2}, \cdots, \Phi(X)\left(g_{s} Y_{s}\right)\right) \\
=\left(g_{1} g_{2} \cdots g_{s}\right)\left\{(F X)\left(\pi\left(Y_{1}, Y_{2}, \cdots, Y_{s}\right)\right)-X\left(\pi\left(F Y_{1}, Y_{2}, \cdots, Y_{s}\right)\right)\right. \\
\left.-\pi\left(\Phi(X) Y_{1}, Y_{2}, \cdots, Y_{s}\right)-\cdots-\pi\left(Y_{1}, Y_{2}, \cdots, \Phi(X) Y_{s}\right)\right\} \\
-g_{1}\left(X g_{2}\right) g_{3} \cdots g_{s}\left(\pi\left(F Y_{1}, Y_{2}, \cdots, Y_{s}\right)-\pi\left(Y_{1}, F Y_{2}, \cdots, Y_{s}\right)\right) \\
-g_{1} g_{2}\left(X g_{3}\right) \cdots g_{s}\left(\pi\left(F Y_{1}, Y_{2}, \cdots, Y_{s}\right)-\pi\left(Y_{1}, Y_{2}, F Y_{3}, \cdots, Y_{s}\right)\right) \\
\quad \cdots \cdots \cdots \cdots \cdots \cdots \cdots \\
-g_{1} g_{2} \cdots\left(X g_{s}\right)\left(\pi\left(F Y_{1}, Y_{2}, \cdots, Y_{s}\right)-\pi\left(Y_{1}, Y_{2}, \cdots, F Y_{s}\right)\right),
\end{gathered}
$$

where $g_{1}, g_{2}, \cdots, g_{s} \in I_{0}^{0}(M)$. Thus we have

Proposition 7.1. Let $\pi \in \mathscr{I}_{s}^{\circ}(M)$. The expression (7.1) is linear in $X, Y_{1}, Y_{2}$, $\cdots, Y_{s}$ and defines a tensor field $\in \mathscr{I}_{s+1}^{0}(M)$ if and only if the tensor $\pi$ satisfies

$$
\pi\left(F Y_{1}, Y_{2}, \cdots, Y_{s}\right)=\pi\left(Y_{1}, F Y_{2}, \cdots, Y_{s}\right)
$$

$$
=\pi\left(Y_{1}, Y_{2}, \cdots, F Y_{s}\right)
$$

for any $Y_{1}, Y_{2}, \cdots, Y_{s} \in \mathscr{I}_{0}^{1}(M)$.

When $F$ is an almost complex structure in $M$, a tensor field $\pi \in \mathscr{I}_{s}^{0}(M)$ which satisfies (7.3) is said to be pure. Thus we have

CoRollary 7.1. If $\pi$ is a pure tensor field $\in \mathscr{I}_{s}^{0}(M)$ in $M$ with almost complex structure $F$, then (7.1) defines a tensor field $\in \mathscr{I}_{s+1}^{0}(M)$.

When this tensor field vanishes, $\pi$ is said to be almost analytic.

On the other hand, (7.2) shows

$$
C\left(X, Y_{1}, Y_{2}, \cdots, Y_{s}\right)\left[(F X)\left(\pi\left(Y_{1}, Y_{2}, \cdots, Y_{s}\right)\right)-X\left(\pi\left(F Y_{1}, Y_{2}, \cdots, Y_{s}\right)\right)\right.
$$

$$
\begin{aligned}
& -\pi\left(\Phi(X) Y_{1}, Y_{2}, \cdots, Y_{s}\right)-\cdots-\pi\left(Y_{1}, Y_{2}, \cdots, \Phi(X) Y_{s}\right) \\
& \left.+\sum_{t=2}^{s}(-1)^{t-1}\left\{\pi\left(F X, Y_{1}, \cdots,\left[Y_{t-1}, Y_{t}\right], \cdots, Y_{s}\right)-\pi\left(X, Y_{1}, \cdots, F\left[Y_{t-1}, Y_{t}\right], \cdots, Y_{s}\right)\right\}\right]
\end{aligned}
$$

is linear in $X, Y_{1}, \cdots, Y_{s}$ and defines a tensor field of type $(0, s+1)$, where $C\left(X, Y_{1}, \cdots, Y_{s}\right)$ denotes the sum of terms obtained by applying a cyclic permutation of $X, Y_{1}, \cdots, Y_{s}$. 
REMARK 7. 1. Let $X^{h}, Y_{1}{ }^{h}, \cdots, Y_{s}{ }^{h}, F_{i}{ }^{h}, \pi_{\jmath_{s} \cdots j_{1}}$ be components of $X, Y_{1}, \cdots, Y_{s}, F, \pi$ with respect to a natural coordinate system in $M$. Then the expression (7.1) has components

$$
\left\{F_{k}^{a} \partial_{a} \pi_{\jmath_{s} \cdots \jmath_{1}}-\partial_{k}\left(\pi_{a \jmath_{s-1} \cdots \jmath_{1}} F_{\jmath_{s}}^{a}\right)+\sum_{t=1}^{s} \pi_{\jmath_{s} \cdots a \cdots j_{1}} \partial_{\jmath_{t}} F_{k}^{a}\right\} X^{k} Y_{1}^{\jmath s} \ldots Y_{s}^{\jmath_{1}}
$$

$$
+\sum_{t=2}^{s}\left(\pi_{j_{s} \cdots a \cdots \jmath_{1}} F_{j_{t}}{ }^{a}-\pi_{a \jmath_{s-1} \cdots \jmath_{1}} F_{\jmath_{s}}{ }^{a}\right) Y_{1}{ }^{\jmath s} \cdots\left(X^{k} \partial_{k} Y_{t}{ }^{\jmath t}\right) \cdots Y_{s}^{\jmath^{1}}
$$

Equation (7.5) shows, as Proposition 7.1 says, that

$$
\begin{gathered}
(F, \pi)_{k j_{s} \cdots j_{1}}=F_{k}^{a} \partial_{a} \pi_{j_{s} \cdots j_{1}}-\partial_{k}\left(\pi_{a j_{s-1} \cdots j_{1}} F_{\jmath s}{ }^{a}\right) \\
+\sum_{t=1}^{s} \pi_{j_{s} \cdots a \cdots j_{1}} \partial_{j_{t}} F_{k}{ }^{a}
\end{gathered}
$$

are components of a tensor field of type $(0, s+1)$ when the conditions

$$
\pi_{\jmath \cdots a \cdots \jmath_{1}} F_{\jmath_{t}}{ }^{a}=\pi_{a \jmath_{s-1} \cdots \jmath_{1}} F_{\jmath_{s}}{ }^{a} \quad(t=1,2, \cdots, s-1)
$$

are satisfied. But (7.5) shows also that

$$
(s+1)(F, \pi)_{\left[k j_{s} \cdots j_{1}\right]}
$$

are components of a tensor field of type $(0, s+1)$, skew-symmetric in all indices, without the condition (7.2).

In the notation of Frölicher and Nijenhuis, we have

$$
(s+1)(F, \pi)_{\left[k_{\left.\jmath_{s} \cdots j_{1}\right]}\right]}=[F, \pi]_{k_{\jmath_{s} \cdots \jmath_{1}}}-[I, \pi \circ F]_{k \jmath_{s} \cdots \jmath_{1}},
$$

if $\pi$ is skew-symmetric.

\section{§ 8. Operator $\Psi s(X, Y)$ applied to a vector field.}

We next define an operator $\Psi s(X, Y)$ or simply $\Psi(X, Y)$, associated with a fixed $S \in \mathscr{I}_{2}^{1}(M)$ and arbitrary $X, Y \in \mathscr{I}_{0}^{1}(M)$, which is applied to an arbitrary $Z \in \mathscr{I}_{0}^{1}(M)$ and gives a vector field

$$
\Psi(X, Y) Z=-\left(\mathcal{L}_{Z} S\right)(X, Y)
$$

that is,

$$
\Psi(X, Y) Z=[S(X, Y), Z]-S([X, Z], Y)-S(X,[Y, Z]) .
$$

We see from (8.1) that $\Psi(X, Y) Z$ is linear in $X$ and $Y$, but not in $Z$ in general. We have in fact

(8. 3) $\Psi(X, Y)(h Z)=h(\Psi(X, Y) Z)+((S(X, Y)) h) Z-(X h) S(Z, Y)-(Y h) S(X, Z)$

for any $h \in \mathscr{I}_{0}^{0}(M)$. 
§9. Operator $\Psi(X, Y)$ applied to a tensor field of type $(1,1)$.

Let $F \in \mathscr{I}_{1}^{1}(M)$. We consider the expression

$$
\Psi(X, Y)(F Z)-F(\Psi(X, Y) Z)=\left(-\mathcal{L}_{F Z} S+F \mathcal{L}_{Z} S\right)(X, Y)
$$

that is,

$$
\begin{aligned}
& \Psi(X, Y)(F Z)-F(\Psi(X, Y) Z) \\
= & {[S(X, Y), F Z]-S([X, F Z], Y)-S(X,[Y, F Z]) } \\
& -F\{[S(X, Y), Z]-S([X, Z], Y)-S(X,[Y, Z])\} .
\end{aligned}
$$

By the definition, (9.1) is linear in $X$ and $Y$ but not in $Z$ in general. We have in fact

$$
\begin{aligned}
& \Psi(X, Y)(F(h Z))-F(\Psi(X, Y)(h Z)) \\
= & h\{\Psi(X, Y)(F Z)-F(\Psi(X, Y) Z)\} \\
& -(X h)(S(F Z, Y)-F(S(Z, Y)))-(Y h)(S(X, F Z)-F(S(X, Z))) .
\end{aligned}
$$

Thus we have

Proposition 9.1. The expression (9.2) defines a tensor field of type $(1,3)$ if and only if $F$ satisfies

$$
F(S(Y, Z))=S(F Y, Z)=S(Y, F Z) .
$$

Thus if (9.4) are satisfied, we put

$$
\begin{aligned}
& \Psi(X, Y)(F Z)-F(\Psi(X, Y) Z) \\
= & (\Psi(X, Y) F) Z=(\Psi F)(X, Y, Z) .
\end{aligned}
$$

If (9.4) are satisfied, then (3.2) can be written as

$$
\begin{aligned}
& (\Phi(X) S)(Y, Z) \\
= & {[F X, S(Y, Z)]-F[X, S(Y, Z)]-S([F X, Y], Z) } \\
& +F(S([X, Y], Z))-S(Y,[F X, Z])+F(S(Y,[X, Z])) .
\end{aligned}
$$

Thus (9.2) and (9.6) show that

$$
(\Phi(X) S)(Y, Z)+(\Psi(Y, Z) F) X=0,
$$

when (9.4) are satisfied.

Coming back to the general case, we have from (9.3) 


$$
\begin{aligned}
& \Psi(f X, g Y)(F(h Z))-F(\Psi(f X, g Y)(h Z)) \\
= & f g h\{\Psi(X, Y)(F Z)-F(\Psi(X, Y) Z)\}-f g(X h) S(F Z, Y) \\
& -f g(Y h) S(X, F Z)+f g(X h) F(S(Z, Y))+f g(Y h) F(S(X, Z)),
\end{aligned}
$$

from which

$$
\begin{gathered}
\Psi(f X, g Y)(F(h Z))-F(\Psi(f X, g Y)(h Z))+\Psi(g Y, h Z)(F(f X)) \\
-F(\Psi(g Y, h Z)(f X))+\Psi(h Z, f X)(F(g Y))-F(\Psi(h Z, f X)(g Y)) \\
=f g h\{\Psi(X, Y)(F Z)-F(\Psi(X, Y) Z)+\Psi(Y, Z)(F X) \\
\quad-F(\Psi(Y, Z) X)+\Psi(Z, X)(F Y)-F(\Psi(Z, X) Y)\} \\
-f g(X h) S(F Z, Y)-f g(Y h) S(X, F Z)+f g(X h) F(S(Z, Y)) \\
+f g(Y h) F(S(X, Z))-g h(Y f) S(F X, Z)-g h(Z f) S(Y, F X) \\
+g h(Y f) F(S(X, Z))+g h(Z f) F(S(Y, X))-h f(Z g) S(F Y, X) \\
-h f(X g) S(Z, F Y)+h f(Z g) F(S(Y, X))+h f(X g) F(S(Z, Y)) .
\end{gathered}
$$

Forming (3.6)-(9.8), we obtain

$$
\begin{aligned}
& \Phi(f X)(S(g Y, h Z))-S(\Phi(f X)(g Y), h Z)-S(g Y, \Phi(f X)(h Z)) \\
& +\Phi(g Y)(S(h Z, f X))-S(\Phi(g Y)(h Z), f X)-S(h Z, \Phi(g Y)(f X)) \\
& +\Phi(h Z)(S(f X, g Y))-S(\Phi(h Z)(f X), g Y)-S(f X, \Phi(h Z)(g Y)) \\
& -\Psi(f X, g Y)(F(h Z))-F(\Psi(f X, g Y)(h Z)) \\
& -\Psi(g Y, h Z)(F(f X))-F(\Psi(g Y, h Z)(f X)) \\
& -\Psi(h Z, f X)(F(g Y))-F(\Psi(h Z, f X)(G Y)) \\
& =f g h\{\Phi(X)(S(Y, Z))-S(\Phi(X) Y, Z)-S(Y, \Phi(X) Z) \\
& \quad+\Phi(Y)(S(Z, X))-S(\Phi(Y) Z, X)-S(Z, \Phi(Y) X) \\
& \quad+\Phi(Z)(S(X, Y))-S(\Phi(Z) X, Y)-S(X, \Phi(Z) Y) \\
& -\Psi(X, Y)(F Z)-F(\Psi(X, Y) Z) \\
& -\Psi(Y, Z)(F X)-F(\Psi(Y, Z) X) \\
& -\Psi(Z, X)(F Y)-F(\Psi(Z, X) Y)\}
\end{aligned}
$$

which shows that 


$$
C(X, Y, Z)[\Phi(X)(S(Y, Z))-S(\Phi(X) Y, Z)-S(Y, \Phi(X) Z)
$$

$$
-\Psi(X, Y)(F Z)-F(\Psi(X, Y) Z)]
$$

gives a tensor of type $(1,3)$, where $C(X, Y, Z)[\quad]$ means the sum of terms obtained by a cyclic permutation of $X, Y$, and $Z$.

Remark 9. 1. The expression (9.2) has components with respect to a natural coordinate system in $M$,

$$
\begin{aligned}
\left(-F_{k}{ }^{a} \partial_{a} S_{j i}{ }^{h}-S_{a i}{ }^{h} \partial_{j} F_{k}{ }^{a}-S_{j a}{ }^{h} \partial_{i}{F_{k}}^{a}+S_{j i}{ }^{a} \partial_{a} F_{k}{ }^{h}+F_{a}{ }^{h} \partial_{k} S_{j i}{ }^{a}\right) Z^{k} Y^{j} X^{\imath} \\
+\left(F_{a}{ }^{h} S_{k \imath}{ }^{a}-S_{a i}{ }^{h} F_{k}{ }^{a}\right)\left(\partial_{j} Z^{k}\right) Y^{j} X^{\imath}+\left(F_{a}{ }^{h} S_{j k}{ }^{a}-S_{j a}{ }^{h}{F_{k}}^{a}\right)\left(\partial_{i} Z^{k}\right) Y^{j} X^{\imath} .
\end{aligned}
$$

Equation (9.10) shows, as Proposition 9.1 says, that

$$
(S, F)_{k j i}{ }^{h}=-F_{k}^{a} \partial_{a} S_{j i}{ }^{h}-S_{a i}{ }^{h} \partial_{j} F_{k}{ }^{a}-S_{j a}{ }^{h} \partial_{i} F_{k}{ }^{a}+S_{j i}{ }^{a} \partial_{a} F_{k}{ }^{h}+F_{a}{ }^{h} \partial_{k} S_{j i}{ }^{a}
$$

are components of a tensor field of type $(1,3)$ when the conditions

$$
F_{a}{ }^{h} S_{k i}{ }^{a}=F_{k}{ }^{a} S_{a i}{ }^{h}=F_{i}{ }^{a} S_{k a}{ }^{h}
$$

are satisfied.

But (3.10) shows,

$$
(F, S)_{k j i}{ }^{h}+(S, F)_{k j i}^{h}=0 .
$$

Thus, when the tensor $S_{j i}^{h}$ is a vector-valued 2 -form, the tensor (9.9) is equal to $2[F, S]$.

\section{$\S 10$. Operator $\Psi(X, Y)$ applied to a tensor field of type $(1, s)$.}

Let $T \in \mathscr{I}_{s}^{1}(M)$. We consider the expression

$$
\begin{aligned}
= & \left(-\mathcal{L}_{T\left(Z_{1}, Z_{2}, \cdots, Z_{s}\right)} S\right)(X, Y) \\
& +T\left(\left(\mathcal{L}_{Z_{1}} S\right)(X, Y), Z_{2}, \cdots, Z_{s}\right)+\cdots+T\left(Z_{1}, Z_{2}, \cdots,\left(\mathcal{L}_{Z_{s}} S\right)(X, Y)\right),
\end{aligned}
$$

that is,

$$
\begin{aligned}
& \Psi(X, Y)\left(T\left(Z_{1}, Z_{2}, \cdots, Z_{s}\right)\right)-T\left(\Psi(X, Y) Z_{1}, Z_{2}, \cdots, Z_{s}\right)-\cdots-T\left(Z_{1}, Z_{2}, \cdots, \Psi(X, Y) Z_{s}\right) \\
= & {\left[S(X, Y), T\left(Z_{1}, Z_{2}, \cdots, Z_{s}\right)\right]-S\left(\left[X, T\left(Z_{1}, Z_{2}, \cdots, Z_{s}\right)\right], Y\right)-S\left(X,\left[Y, T\left(Z_{1}, Z_{2}, \cdots, Z_{s}\right)\right]\right) } \\
(10.2) \quad & -T\left(\left[S(X, Y), Z_{1}\right]-S\left(\left[X, Z_{1}\right], Y\right)-S\left(X,\left[Y, Z_{1}\right]\right), Z_{2}, \cdots, Z_{s}\right) \\
& -\cdots \\
& -T\left(Z_{1}, Z_{2}, \cdots,\left[S(X, Y), Z_{s}\right]-S\left(\left[X, Z_{s}\right], Y\right)-S\left(X,\left[Y, Z_{s}\right]\right)\right) .
\end{aligned}
$$


We see from (10.1) that

$$
\begin{aligned}
\Psi(X, Y)\left(T\left(Z_{1}, Z_{2}, \cdots, Z_{s}\right)\right) \\
\quad-T\left(\Psi(X, Y) Z_{1}, \cdots, Z_{s}\right)-\cdots-T\left(Z_{1}, Z_{2}, \cdots, \Psi(X, Y) Z_{s}\right)
\end{aligned}
$$

is linear in $X$ and $Y$, but not in $Z_{1}, Z_{2}, \cdots, Z_{s}$ in general. We have in fact

$$
\begin{aligned}
& \Psi(X, Y)\left(T\left(h_{1} Z_{1}, h_{2} Z_{2}, \cdots, h_{s} Z_{s}\right)\right) \\
& \quad-T\left(\Psi(X, Y)\left(h_{1} Z_{1}\right), h_{2} Z_{2}, \cdots, h_{s} Z_{s}\right)-\cdots-T\left(h_{1} Z_{1}, h_{2} Z_{2}, \cdots, \Psi(X, Y)\left(h_{s} Z_{s}\right)\right) \\
& =\left(h_{1} h_{2} \cdots h_{s}\right)\left\{\Psi(X, Y)\left(T\left(Z_{1}, Z_{2}, \cdots, Z_{s}\right)\right)\right. \\
& \left.\quad-T\left(\Psi(X, Y) Z_{1}, Z_{2}, \cdots, Z_{s}\right)-\cdots-T\left(Z_{1}, Z_{2}, \cdots, \Psi(X, Y) Z_{s}\right)\right\} \\
& \quad-\left(X h_{1}\right) h_{2} \cdots h_{s}\left(S\left(T\left(Z_{1}, Z_{2}, \cdots, Z_{s}\right), Y\right)-T\left(S\left(Z_{1}, Y\right), Z_{2}, \cdots, Z_{s}\right)\right) \\
& -h_{1}\left(X h_{2}\right) \cdots h_{s}\left(S\left(T\left(Z_{1}, Z_{2}, \cdots, Z_{s}\right), Y\right)-T\left(Z_{1}, S\left(Z_{2}, Y\right), \cdots, Z_{s}\right)\right) \\
& \quad \cdots \\
& -h_{1} h_{2} \cdots\left(X h_{s}\right)\left(S\left(T\left(Z_{1}, Z_{2}, \cdots, Z_{s}\right), Y\right)-T\left(Z_{1}, Z_{2}, \cdots, S\left(Z_{s}, Y\right)\right)\right. \\
& -\left(Y h_{1}\right) h_{2} \cdots h_{s}\left(S\left(X, T\left(Z_{1}, Z_{2}, \cdots, Z_{s}\right)\right)-T\left(S\left(X, Z_{1}\right), Z_{2}, \cdots, Z_{s}\right)\right) \\
& -h_{1}\left(Y h_{2}\right) \cdots h_{s}\left(S\left(X, T\left(Z_{1}, Z_{2}, \cdots, Z_{s}\right)\right)-T\left(Z_{1}, S\left(X, Z_{2}\right) \cdots Z_{s}\right)\right) \\
& \cdots \\
& -h_{1} h_{2} \cdots\left(Y h_{s}\right)\left(S\left(X, T\left(Z_{1}, Z_{2}, \cdots, Z_{s}\right)\right)-T\left(Z_{1}, Z_{2}, \cdots, S\left(X, Y_{s}\right)\right) .\right.
\end{aligned}
$$

Thus we have

Proposition 10.1. The expression (10.1) is linear in $X, Y, Z_{1}, \cdots, Z_{s}$ and defines a tensor field of type $(1, s+2)$ if and only if $T$ satisfies

$$
\begin{aligned}
S\left(T\left(Z_{1}, Z_{2}, \cdots, Z_{s}\right), Y\right) & =T\left(Z_{1}, \cdots, S\left(Z_{t}, Y\right), \cdots, Z_{s}\right), \\
S\left(X, T\left(Z_{1}, Z_{2}, \cdots, Z_{s}\right)\right) & =T\left(Z_{1}, \cdots, S\left(X, Z_{t}\right), \cdots, Z_{s}\right) \\
(1 \leqq t \leqq s) . &
\end{aligned}
$$

REMARK 10.1. The expression (10.1) has following components with respect to a natural coordinate system in $M$.

$$
\begin{aligned}
& \left(S_{k j}{ }^{a} \partial_{a} T_{\imath_{s} \cdots i_{1}}{ }^{h}-T_{i_{s} \cdots i_{1}}{ }^{a} \partial_{a} S_{k j}{ }^{h}-S_{a j}{ }^{h} \partial_{k} T_{\imath_{s} \cdots i_{1}}{ }^{a}-S_{k a}{ }^{h} \partial_{j} T_{i_{s} \cdots i_{1}}{ }^{a}\right. \\
& \left.+\sum_{t=1}^{s} T_{i_{s} \ldots a i_{1}}{ }^{n} \partial_{i_{t}} S_{\jmath}{ }^{a}\right) X^{k} Y^{j} Z_{1}{ }^{\imath s} \ldots Z_{s}^{i 1} \\
& +\sum_{t=1}^{s}\left(T_{i_{s} \cdots a i_{1}}{ }^{h} S_{i_{t}}{ }^{a}-S_{a j}{ }^{h} T_{i_{s} \cdots i_{1}}{ }^{a}\right) Y^{j} Z_{1}{ }^{i s \cdots(}\left(X^{k} \partial_{k} Z^{i t}\right) \cdots Z_{s}{ }^{i 1} \\
& +\sum_{t=1}^{s}\left(T_{i_{s} \cdots \cdots \cdots i_{1}}{ }^{h} S_{k i_{t}}{ }^{a}-S_{k a}{ }^{h} T_{\imath_{s} \cdots i_{1}}{ }^{a}\right) X^{k} Z_{1}{ }^{2 s \cdots}\left(Y^{j} \partial_{j} Z^{i t}\right) \cdots Z_{s}^{i 1} .
\end{aligned}
$$


This equation shows, as Proposition 10.1 says, that

$$
\begin{aligned}
(S, T)_{k j i_{s} \cdots i_{1}}{ }^{n}= & S_{k j}{ }^{a} \partial_{a} T_{i_{s} \cdots i_{1}}{ }^{h}-T_{i_{s} \cdots i_{1}}{ }^{a} \partial_{a} S_{k j}{ }^{h} \\
& -S_{a j}{ }^{h} \partial_{k} T_{i_{s} \cdots i_{1}}{ }^{a}-S_{k a}{ }^{h} \partial_{j} T_{i^{\prime} \cdots i_{1}}{ }^{a}+\sum_{t=1}^{s} T_{i_{s} \cdots a \cdots i_{1}}{ }^{h} \partial_{i_{t}} S_{k j}{ }^{a}
\end{aligned}
$$

are components of a tensor field of type $(1, s+2)$ when the conditions

$$
\begin{aligned}
& T_{i_{s} \cdots i_{1}}{ }^{a} S_{a j}{ }^{h}=T_{\imath_{s} \cdots a \cdots i_{1}}{ }^{h} S_{i_{t} t^{\prime}}{ }^{a}, \\
& T_{\imath_{s} \cdots i_{1}}{ }^{a} S_{k a}{ }^{h}=T_{i_{s} \cdots \cdots \cdots i_{1}}{ }^{h} S_{k v_{t}}{ }^{a}
\end{aligned}
$$

are satisfied.

If $S$ and $T$ are both skew-symmetric, we can show that

$$
(s+2)(S, T)_{\left[k j i_{s} \cdots i_{1}\right]^{h}}
$$

are components of a tensor field without condition (10.2). Moreover we have, by a straightforward computation,

$$
(s+1)(s+2)(S, T)_{\left[k_{j i} i^{\left.\cdots \cdots i_{1}\right]}\right.}{ }^{h}=2[S, T]_{k j i_{s} \cdots i_{1}}{ }^{h}
$$

in Nijenhuis' notation.

\section{§11. Operator $\Psi(X, Y)$ applied to a 1-form.}

In this and next sections, we always assume that the $S$ is a vector-valued 2 -form.

Let $\omega \in \mathscr{I}_{1}^{0}(M)$. We consider the expression

$$
\begin{aligned}
& (S(X, Y))(\omega(Z))-X(\omega(S(Z, Y)))-Y(\omega(S(X, Z))) \\
& -\omega(S([X, Y], Z))-\omega(\Psi(X, Y) Z) \\
= & \left(\mathcal{L}_{S(X, Y)} \omega\right)(Z)-\left(\mathcal{L}_{X}(\omega \circ S)\right)(Z, Y) \\
& -\left(\mathcal{L}_{Y}(\omega \circ S)\right)(X, Z)+(\omega \circ S)([X, Y], Z),
\end{aligned}
$$

where $X, Y, Z \in \mathscr{I}_{0}^{1}(M)$ and the tensor $\omega^{\circ} S$ is defined by

$$
(\omega \circ S)(X, Y)=\omega(S(X, Y))
$$

for any $X, Y \in \mathscr{I}_{0}^{1}(M)$.

Since we have

$$
\begin{aligned}
& (f X)(\omega(S(Z, g Y)))-(g Y)(\omega(S(f X, Z)))-(S([f X, g Y], Z)) \\
= & (f g)\{X \omega(S(Z, Y))-Y(\omega(S(X, Z)))-\omega(S([X, Y], Z))\},
\end{aligned}
$$

we see that (11.1) is linear in $X$ and $Y$, 
We see also that (11.1) is linear in $Z$. Thus we have

Proposition 11. 1. The expression (11.1) is linear in $X, Y, Z$ and defines $a$ tensor field of type $(0,3)$.

We sometimes denote this tensor field by

$$
(\Psi(X, Y) \omega)(Z)=(\Psi \omega)(X, Y, Z) .
$$

REMARK 11.1. The expression (11.1) has following components with respect to a natural coordinate system

$$
(S, \omega)_{k j i}=S_{k \jmath}{ }^{a} \partial_{a} \omega_{i}-\partial_{k}\left(S_{i j}{ }^{a} \omega_{a}\right)-\partial_{j}\left(S_{k \imath}{ }^{a} \omega_{a}\right)+\left(\partial_{i} S_{k \jmath}{ }^{a}\right) \omega_{a},
$$

from which we have,

$$
3(S, \omega)_{[k j i]}=[S, \omega]_{k j i}-2[I, \omega \circ S]_{k j i}
$$

in the notation of Frölicher and Nijenhuis.

\section{$\S 12$. Operator $\Psi(X, Y)$ applied to a tensor field of type $(0, s)$.}

Let $\pi \in \mathscr{I}_{s}^{0}(M)$. We consider the expression

$$
\begin{aligned}
& \left(\mathcal{L}_{S(X, Y)} \pi\right)\left(Z_{1}, \cdots, Z_{s}\right)-\left(\mathcal{L}_{X}(\pi \circ S)\right)\left(Z_{1}, Y, Z_{2}, \cdots, Z_{s}\right) \\
& \quad-\left(\mathcal{L}_{Y}(\pi \circ S)\right)\left(X, Z_{1}, Z_{2}, \cdots, Z_{s}\right)+(\pi \circ S)\left([X, Y], Z_{1}, Z_{2}, \cdots, Z_{s}\right),
\end{aligned}
$$

where

$$
(\pi \circ S)\left(X, Z_{1}, Z_{2}, \cdots, Z_{s}\right)=\pi\left(S\left(X, Z_{1}\right), Z_{2}, \cdots, Z_{s}\right) .
$$

This expression defines a tensor field of type $(0, s+2)$ if and only if

$$
\begin{aligned}
& \pi\left(Z_{1}, \cdots, S(X, Y), \cdots, Z_{s}\right) \\
= & \pi\left(S\left(Z_{1}, Y\right), Z_{2}, \cdots, X, \cdots, Z_{s}\right) \\
= & \pi\left(S\left(X, Z_{1}\right), Z_{2}, \cdots, Y_{1}, \cdots, Z_{s}\right) .
\end{aligned}
$$

REMARK 12.1. Expression (12.1) has local components

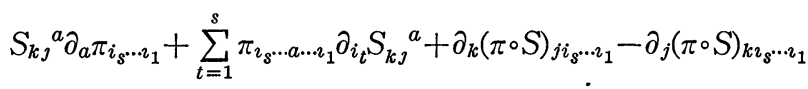

$$
\begin{aligned}
& -\sum_{t=2}^{s}\left(\pi_{i_{s} \cdots a \cdots i_{1}} S_{k j}{ }^{a}-S_{k i_{s}}{ }^{a} \partial_{a} \pi_{i_{s-1} \cdots \jmath \cdots i_{1}}\right) X^{k}\left(\partial_{i_{t}} Y^{j}\right) \\
& -\sum_{t=3}^{s}\left(\pi_{\imath_{s} \cdots \cdots \cdots i_{1}} S_{k \jmath}{ }^{a}-S_{i_{s} \jmath}{ }^{a} \partial_{a} \pi_{\imath_{\xi-1} \cdots k \cdots i_{7}}\right)\left(\partial_{i_{t}} X^{k}\right) Y^{j},
\end{aligned}
$$


So

$$
\begin{aligned}
(S, \pi)_{k j i_{s} \cdots i_{1}}= & S_{k \jmath}{ }^{a} \partial_{a} \pi_{\imath_{s} \cdots i_{1}}+\sum_{t=1}^{s} \pi_{\imath_{s} \cdots \cdots \cdots i_{1}} \partial_{i_{t}} S_{k j}{ }^{a} \\
& +\partial_{k}(\pi \circ S)_{j i_{s} \cdots i_{1}}-\partial_{j}(\pi \circ S)_{k r_{s} \cdots i_{1}}
\end{aligned}
$$

are components of a tensor field of type $(0, s+2)$ under the condition (12.2).

We have

$$
(s+1)(s+2)(S, \pi)_{\left[k j i_{s} \cdots i_{1}\right]}=2[S, \pi]_{k j i_{s} \cdots i_{1}}+2(s+1)[I, \pi \circ S]_{k j i_{s} \cdots i_{1}}
$$

in the notation of Frölicher and Nijenhuis.

\section{BIBLIOGRAPHY}

[1] Frölicher, A., ANd A. NiJenhuis, Theory of vector-valued forms, I; II. Proc. Kon. Ned. Akad. Wet. Amsterdam, A 59 (1956), 339-350 ; 351-359.

[2] FRölicher, A., AND A. NiJenhuis, Some new cohomology invariants for complex manifolds, I; II. ibid. 59 (1956), 540-552; 553-564.

[ 3 ] Kobayashi, S., and K. Nomizu, Foundations of differential geometry. Interscience Tract, No. 15 (1963).

[4] Newlander, A., And L. Nirenberg, Complex analytic coordinates in almost complex manifolds. Ann. of Math. 65 (1957), 391-404.

[5] NiJenhuis, A., $X_{n-1}$-forming sets of eigenvectors. Indag. Math. 13 (1951), 200222.

[6] Nijenhuis, A., Jacobi-type identities for bilinear differential concomitants of certain tensor fields, I; II. Proc. Kon. Ned. Akad. Wet. Amsterdam, A 58 (1955), 390-397; 398-403.

[7] SAtô, I., Almost analytic tensor fields in almost complex manifolds. Tensor 17 (1966), 105-119.

[8] Sawaki, S., And K. Takamatsu, A characterization of almost analytic tensor fields in almost complex manifolds. Sci. Rep. of Niigata Univ. 5 (1967), 1-6.

[9] Schouten, J. A., Ricci-Calculus. Springer (1954).

[10] ŚLebodzińsKi, W., Contribution à la géométrie différentielle d'un tenseur mixte de valence deux. Colloq. Math. 13 (1964), 49-54.

[11] Tachibana, S., Analytic tensor and its generalization. Tôhoku Math. J. 12 (1960), 208-221.

[12] Tachibana, S., AND S. Kotō, On almost analytic functions, tensors and invariant subspaces. Tôhoku Math. J. 14 (1962), 177-186.

[13] Walker, A. G., Dérivation torsionnelle et seconde torsion pour une structure presque complexe. C. R. Acad. Sci. Paris 245 (1957), 1213-1215.

[14] Willmore, T. J., Derivation and vector 1-form. J. London Math. Soc. 35 (1960), 425-432.

[15] WillmoRe, T. J., Note on Ślebodziński tensor of an almost complex structure. J. London Math. Soc. 43 (1968) 321-322.

[16] YANO, K., Differential geometry on complex and almost complex spaces. Pergamon Press (1965). 
[17] Yano, K., AND M. AKo, Almost analytic vectors in almost complex spaces. Tôhoku Math. J. 13 (1961), 24-45.

[18] YANO, K., AND M. AKo, Notes on covariant almost analytic vector fields. Tôhoku Math. J. 19 (1967), 187-197.

[19] Yano, K., AND T. Nagano, On geodesic vector fields in a compact orientable Riemannian space. Comment. Math. Helv. 35 (1961), 55-64.

Department of Mathematics,

Tokyo Institute of TeChNOLOGy. 